\title{
Cosmopolitismo kantiano: una propuesta racional pragmática frente al sentimentalismo de Richard Rorty. Acerca de cómo educar para respetar efectivamente a las personas y a las diferentes culturas en materia de derechos humanos
}

Héctor Pérez Guido

UNIVERSIDAD IBEROAMERICANA

\section{Resumen:}

En las siguientes líneas quiero presentar una exposición en contra del argumento de Richard Rorty a favor del sentimentalismo como forma de educación en los derechos humanos. Para ello, me basaré en el concepto de ciudadano del mundo de Immanuel Kant, tomando en cuenta diversas obras de su autoría, pero sobre todo su Antropología en sentido pragmático $(\mathrm{ApH})$; después, concluiré algunas implicaciones acompañando mi argumento con algunas miradas actuales sobre este concepto en Martha Nussbaum y Anthony Appiah.

Palabras clave: Kant, Rorty, educación, cosmopolitismo, Antropología en sentido pragmático.

\section{Abstract:}

In the following lines I want to present an exposition against Richard Rorty's argument in favor of sentimentality as a form of education in human rights. To do this, I will build on Immanuel Kant's concept of the world citizen, taking into account various works of his authorship, but mainly his Anthropology in a pragmatic sense (ApH); later, I will conclude some implications accompanying my argument with some current views on this concept in Martha Nussbaum and Anthony Appiah.

KEYWORDS: Kant, Rorty, education, cosmopolitism, Anthropology in a pragmatic sense. 
En un texto de los años 90, el filósofo norteamericano Richard Rorty trata de convencernos de que, en cuestión de practicar los derechos humanos, es mejor que leamos novelas como La cabaña del tio Tom en lugar de tratados filosóficos como la Fundamentación de la metafísica de las costumbres (Rorty 2000, 240). Según Rorty, en la actualidad existe una "cultura de los derechos humanos" que ha ganado más adeptos a través de los sentimientos que con razones o fundamentos. ${ }^{1}$ Las historias largas y tristes que nos han contado los novelistas y cronistas de guerra acerca de lo que ocurre cuando los derechos humanos son violados, a decir del norteamericano, han provocado un mayor efecto en las personas que creen en estos derechos que el que habrían de producir las teorías filosóficas dedicadas a mostrar que debemos respetarnos debido a la existencia de una naturaleza o esencia humana que nos hace iguales. La racionalidad, desde esta perspectiva, está pasada de moda y, por tanto, debería ser desechada en los argumentos acerca de los derechos humanos; en su lugar, dice Rorty, la manipulación emocional es una mejor manera de hacer que los ricos y poderosos, quienes gozan de las bondades del progreso (riqueza, educación y ocio), sientan ganas de preocuparse por aquellos que, de acuerdo con esta visión, se encuentran desposeídos de seguridad y de simpatía (Rorty 2000, 226).

En resumen, el diagnóstico de Rorty es el siguiente: vivimos en un mundo donde algunas sociedades se vieron beneficiadas por la historia con la Revolución francesa y el fin del comercio transatlántico de esclavos, ya que se volvieron ricas, educadas y gozan del tiempo para preocuparse por quienes no tienen estos beneficios; de ahí que hayan adoptado ideales ilustrados como el de los derechos humanos (Rorty 2000, 230). Estas personas que en la actualidad viven en condiciones favorables se sienten más dispuestas a dar ayuda y tolerar a otras cuando se conmueven por las narraciones que los escritores y reporteros les cuentan sobre la situación de la gente que vive en lugares donde no tienen las mismas condiciones privilegiadas que ellos. Con esto, las personas favorecidas logran incluso dejar de sentir rechazo hacia las diferencias con las otras, en virtud de que entienden que éstas prácticas que les parecen deleznables son

1 Rorty retoma este concepto de "cultura de los derechos humanos" del jurista Eduardo Rabossi, quien afirma que estos son ya una práctica y, por ello, no necesitan ya fundamentarse o naturalizarse (Rorty 2000, 223). 
sólo un producto de su situación desventajosa. Otro factor importante a considerar es el triunfo de las ideas del darwinismo acerca de la maleabilidad de los seres humanos, su capacidad de adaptación y, por ende, de ser distintos y progresar, por encima de los fundamentalismos filosóficos que buscaban explicar algo permanente y eterno en el ser humano (Rorty 2000, 228).

Por su parte, los escritos de la filosofía se han orientado a encontrar un fundamento para considerar la igualdad entre las personas, por lo que, de acuerdo con esta tesis, dejan de lado las diferencias culturales y, con ello, no convencen a los individuos para que sientan respeto por otros a pesar de sus diferencias, en tanto que el principio o fundamento al que aluden dichas teorías radica en algo que no es visible para nadie (llámese naturaleza, dignidad o racionalidad). En consecuencia, las personas no encuentran en esos discursos ningún motivo para ayudar, cuidar, proteger o simpatizar con quienes no se les parecen en algo o en nada según su cultura. Dicho de otra manera, los escritos filosóficos no sentimentalistas orientados a demostrar que aparte de las diferencias culturales hay algo idéntico en todas las personas no son eficaces porque no consiguen conmover a la gente para que simpaticen con las diferencias de otros y, por lo tanto, es mejor abandonarlos. Por el contrario, los escritos sentimentalistas son efectivos porque muestran que todos tenemos padres, madres, hijos, hermanos, personas a las que queremos y que por eso habría que respetarnos siempre a pesar de nuestras diferencias.

Hasta aquí, Rorty dice sentirse dentro del relativismo cultural y el irracionalismo, aunque, ciertamente, también mantiene una postura pragmática y con una fuerte base empirista. En cuanto a su pragmatismo, me parece suficiente seńalar la importancia que tiene para el autor medir la efectividad de los discursos sentimentalistas por sobre los racionalistas como principal criterio de verdad. Además, como puede verse, Rorty parece coincidir con la concepción moral de David Hume o Adam Smith, quienes sostenían que el origen de nuestras costumbres se encontraba en nuestra emotividad, esto es, en nuestra capacidad de sentir agrado o desagrado por algo. Parece, entonces, que Rorty sigue a Hume cuando señala que las personas que pertenecen a la cultura de los derechos humanos se sienten motivadas a adoptar algunas prácticas a partir de los sentimientos que les provocan los discursos. 
Lo que esta tesis no explica de manera suficiente es ¡cómo fue que los derechos humanos se convirtieron en una práctica antes de habernos conmovido? Queda claro que, según ella, el sentimiento a favor de los derechos humanos es efectivo en la práctica actual; lo que se da por descontado, al no ser eficaz, es poder dar razones a favor de ellos. Quedamos entonces atrapados en un círculo donde las prácticas se apoyan en los sentimientos y los sentimientos en las prácticas, pero no se explica cómo habría un progreso, ya que las razones no intervienen en ningún momento. No obstante, Rorty concede que los discursos fundamentalistas de filósofos como Platón y Kant sirvieron al menos, en otros tiempos, como inspiración de que las cosas podían ser mejores. A pesar de esto, sigue sin entenderse cómo es que las ideas que estos personajes defendían valiéndose de argumentos racionales se habrían podido convertir en prácticas si, por sí mismos, estos discursos no son sentimentalmente eficaces.

Como es por todos sabido, contrario a la idea de Rorty, Kant no piensa en que las cuestiones morales recaigan en nuestros sentimientos ni, por lo tanto, que hayan de basarse en la naturaleza humana. La idea de Kant es que de la contingencia no puede dar como resultado algo necesario, ${ }^{2}$ por lo cual no es posible sostener que los hábitos y costumbres, que son tan variables y distintos entre sí, puedan llegar a una generalización tan amplia como para considerarlos válidos por siempre. Si volvemos a Hume, como lo hizo Kant, el escocés objeta que todo conocimiento parte de la experiencia sensible y, por lo tanto, no es posible ningún conocimiento a priori, por ello cuando hablamos de causalidad y necesidad nos referimos simplemente al hábito que hemos adquirido en la repetición de nuestras observaciones sobre un efecto de la realidad (Ak. $\mathrm{V}, 50 \mathrm{~s}$ ); en otras palabras, es un uso de palabras que no se refiere a nada que podamos conocer, por lo que habría que descartarlo. En esto, el argumento de Rorty coincide cuando sostiene que la idea de

2 Ak V 12: Es una verdadera contradicción querer sacar la necesidad de una proposición empírica (ex pumice aquam), y con esta necesidad querer proporcionar un juicio de universalidad [...] Sustituir la necesidad subjetiva, es decir el hábito, por la necesidad objetiva que sólo existe en los juicios a priori, quiere decir negar a la razón la facultad de juzgar el objeto, es decir, de conocer un objeto [...] lo cual equivale en el fondo a rechazar el concepto de causa como falso y como un mero engańo del pensamiento. 
naturaleza humana o racionalidad no se refieren a nada visible y, por lo tanto, no producen ningún sentimiento en las personas cuando se les trata de enseñar a respetarlas.

Por su parte, Kant nos dice que "la crítica de la razón práctica en general [...] debe solamente establecer de modo completo los principios de la posibilidad, la extensión y los límites de la razón práctica sin referencia particular a la naturaleza humana" (Ak. V, 8). La propuesta de Kant está en contra de partir de la experiencia para conocer sobre asuntos de moral. En el lugar donde los empiristas encuentran datos sensibles o efectos, Kant opone los principios a priori de la razón. Tradicionalmente, a la apuesta kantiana se le han enfrentado argumentos escépticos sobre el contenido y la capacidad de motivación de las razones o principios a priori sobre la conducta moral, que por razones de espacio no consideraré aquí (cfr. Wallace 2006). No obstante, es fácil ver que la postura de Rorty coincidiría en que la razón práctica no tiene ningún valor en motivación ni de contenido, pues ambos son nulos en tanto efectos. A pesar de esto, a continuación quiero mostrar que la propuesta kantiana incluye una teoría consistente sobre el respeto entre las personas y que, aunque no depende de los efectos, sino de las razones, no desestima las diferencias culturales ni los sentimientos y puede dar explicaciones sobre los cambios progresivos en materia moral, lo cual no es posible, según mi exposición, en la tesis de Rorty.

La idea del cosmopolitismo en Kant ha aparecido, por lo general, relacionada con una postura política orientada hacia el establecimiento de una liga de naciones que respetan ciertos acuerdos para mantener un estado de paz. En la Metafísica de las costumbres (MS), tales acuerdos se refieren principalmente a la libertad de intercambio económico y entre culturas, por lo cual se habla de un derecho cosmopolita entre las naciones (Ak. VI 352). Ahora bien, en la Idea para una historia desde el punto de vista cosmopolita, Kant arguye que ese régimen permite el desarrollo pleno de las capacidades de los ciudadanos dentro de una sociedad. De entre las capacidades que pueden ser desarrolladas están las morales, para lo cual se requiere de instrucción y de ejercicio del juicio. Sólo de esa manera será posible que el hombre elija seguir principios morales en lugar de dejarse llevar por sus impulsos meramente naturales (Ak. VIII 18-27).

De vuelta a la Ms, en un estado natural, los deseos particulares ponen en conflicto a los individuos que se proponen distintos fines 
acordes con su voluntad subjetiva; no obstante, la razón hace posible el establecimiento de un orden jurídico, el estado de derecho, en el que hay leyes jurídicas que orientan de manera coercitiva a que la conducta del hombre respete lo que la razón manda. Sin embargo, antes están las leyes morales que son, en realidad, el fundamento para las jurídicas, ya que las primeras tienen valor por sí mismas y no por su utilidad (Ak. VI 214). El derecho cosmopolita, podríamos decir, entonces, consiste en un conjunto de leyes jurídicas que buscan seguir los principios morales a priori, lo cual implica el uso práctico de la razón.

No obstante, tal uso práctico contempla también el uso pragmático de nuestra razón, esto es, un uso que considera los fines propios de la razón práctica. Para Kant es posible hacer un uso pragmático de la razón si los fines que se proponen son acordes con los principios prácticos. Cabe recordar que el término pragmático tiene varias acepciones dentro del vocabulario de Kant ( $c f r$. Martiarena 2008, pp. 37-42). De acuerdo con la KrV, las leyes pragmáticas van encaminadas a la unidad de los fines desde la libertad, porque pertenecen también al uso práctico de la razón (KrV A 800). En la Ms, se nos dice que "las leyes pragmáticas no se originan propiamente del derecho de los Estados como leyes necesarias, sino de la previsión de una prosperidad general", y que "una historia escrita es pragmática cuando nos hace prudentes, es decir, enseña al mundo cómo puede procurar su provecho mejor [...]" (Ak. IV 417). En un texto más antiguo, "Sobre las diversas razas humanas", con el que presentó sus Lecciones sobre geografía física en 1775, Kant afirma que este curso se trata de un ejercicio en el conocimiento del mundo que "sirve para proporcionar lo pragmático a todas las demás ciencias y habilidades adquiridas de tal manera que se vuelvan útiles, no sólo para la escuela, sino también para la vida, por lo que el aprendiz ya formado es introducido en el escenario de su destino, a saber, en el mundo" (Ak. II 443). Asimismo, hay que tomar en cuenta lo que dice la Antropología en sentido pragmático (ApH) acerca de que el conocimiento pragmático del hombre se diferencia del fisiológico en que éste investiga lo que la naturaleza hace de él, mientras que el pragmático lo que él, como ser que actúa libremente, hace, o puede y debe hacer, de sí mismo (Ak. VII 119). Además, se trata de un conocimiento del hombre como ciudadano del mundo [Weltbürger] (Ak. VII 120). 
De acuerdo con todo lo anterior, podemos decir que el uso pragmático de la razón está dentro del uso práctico, en tanto que se refiere a la libertad de elegir y consiste en buscar unir todos los fines a partir de un principio que es el del destino del hombre, esto es, procurar sacar provecho de todos sus conocimientos para mejorar su propia condición, lo cual lo lleva a convertirse en ciudadano del mundo. Todo lo pragmático, en este sentido, es útil para llegar a ser cosmopolita y, por tanto, podría pensarse que el cosmopolita es, a su vez, pragmático, dado que busca mejorarse a sí mismo. Cabe aclarar y ampliar el sentido en el que se entiende este mejorarse a sí mismo, la misma $\mathrm{ApH}$ nos dice que "el hombre está destinado por su razón a estar en una sociedad con hombres y, en ella, por medio de las artes y las ciencias, a cultivarse, a civilizarse y a moralizarse [...] y a hacerse activamente digno de la humanidad, en lucha con los obstáculos que le depare la rudeza de su propia naturaleza" (Ak. VII 234-235). Queda claro, entonces, que lo pragmático no se restringe a la prosperidad de algunas personas, sino que, de acuerdo con el cosmopolitismo, se refiere a cumplir con aquellas tareas que la razón le impone como necesarias para ser digno de la humanidad, es decir, para corresponder con las obligaciones que tiene toda su especie.

$\mathrm{La} \mathrm{ApH}$, en consecuencia, tiene la finalidad de brindar un tipo de conocimiento útil para que los seres humanos sean cosmopolitas, lo que significa que actúen siempre considerándose tanto a sí mismos como a los otros como fines en sí mismos y nunca como medios, con lo cual se busca cumplir con la exigencia de universalidad en nuestras acciones conforme a la ley moral de la razón práctica: "obra de modo que la máxima de tu voluntad pueda, al mismo tiempo, valer siempre como principio de una legislación universal" (Ak. V 30). Si bien, cumplir a cabalidad con este principio se descubre de inmediato como tarea difícil, o imposible, para un ser racional finito que tiene inclinaciones, la tesis kantiana defiende que eso no le impide tener la aspiración a conseguirlo. De ahí, resulta posible mantenerse en el intento y, por consiguiente, buscar el "progreso al infinito de las propias máximas y de su constancia invariable para un progreso constante", actitud que, en palabras de Kant, corresponde con la virtud, por ser "lo más alto que puede realizar la razón práctica finita” (Ak. V 33). El cosmopolita, en consecuencia, puede ser considerado como aquel que posee la virtud de reconocerse como imperfecto, por lo que asume la tarea de corregir, siempre 
que sea posible, sus máximas para intentar cumplir con la fórmula de la universalidad. En su labor, el ciudadano del mundo no es un mero espectador pasivo ni se deja llevar por el sentido de obligación, sino que examina su juicio, reflexiona y critica, de tal manera que se mantiene autónomo.

En cambio, el sentimentalista descrito por Rorty asume que está en una posición superior dadas las condiciones históricas que le han generado riqueza, educación y ocio, y sólo puede mirar con una simpatía (que puede dar lugar a la condescendencia) hacia los otros. Entonces, para evitar que este tipo de persona condescendiente sea cruel con los desprotegidos hay que contarle historias tristes y conmovedoras que le hagan simpatizar con las diferencias de los otros. Es evidente que este sentimentalista siempre está a expensas de lo que le cuenten los otros, no tiene otra manera de decidir sobre sus actos y carece de un criterio independiente de su contexto para comprender e intentar corregir sus creencias y comportamientos.

"El hombre tiene, pues, que ser educado para el bien" (Ak. VII 325), señala Kant, y para ello está, en este caso, la antropología pragmática. Ésta tiene la finalidad de ofrecer un conocimiento del ciudadano del mundo, como ya hemos visto, y, para ello, se puede valer de medios como "la historia universal, las biografías y hasta las obras de teatro y las novelas" (Ak. VII 121). De tal manera que el conocimiento pragmático no desecha el conocimiento de las culturas ni los textos que tienen la orientación de conmover; por el contrario, son medios adecuados para aumentar el volumen de conocimiento del mundo que conlleva al cosmopolitismo. Así, por ejemplo, el libro tercero de la primera parte de la $\mathrm{ApH}$ trata sobre los afectos y las pasiones. En este sentido, la educación de los sentimientos no queda excluida de la formación del ciudadano del mundo, sino que va acompañada del pensamiento con la finalidad de ejercitar su capacidad de juzgar.

Recordemos la Crítica de la facultad de juzgar: cuando se tiene un caso particular para el cual se busca una regla o ley universal hacemos uso del juicio reflexionante (Ak. V 179). En este aspecto, Carlos Mendiola nos explica que el sensus communis juega un papel importante en este tipo de juicio, pues se encarga de tomar en cuenta a los otros seres humanos y así se posibilita la comunicación, lo cual implica que al hacer uso del juicio reflexionante los seres humanos ya estamos considerando a la humanidad ( $c f r$. Mendio- 
la 2016, pp. 57-69). Por lo tanto, el cosmopolita es aquel que de forma continua está reflexionando sobre su modo de actuar, busca cómo lograr que su máxima pueda convertirse en una ley universal y, para ello, puede partir de su propia experiencia y la de otros. Por eso, Kant considera que dentro de los medios para la formación del ciudadano del mundo están los viajes, pero, antes que nada, "el trato con los conciudadanos o paisanos". Así encontramos que, en su prólogo, la antropología pragmática considera al hombre como alguien que no sólo conoce y contempla al mundo sino como aquel que tiene mundo, esto es, como quien lo ha comprendido y ha entrado en juego con él (Ak. VII 120).

De manera similar, Martha Nussbaum sostiene que "[Kant] defendía una política basada sobre la razón en vez del patriotismo o de un sentimiento grupal, que fuera verdaderamente universal más que comunitaria, activa, reformista y optimista en lugar de conformarse con la contemplación de los horrores [...]" (Nussbaum 2010, 28), lo cual, me parece, coincide en cierta medida con lo que he expuesto. Así mismo, el filósofo contemporáneo Kwame Anthony Appiah ha actualizado el concepto de cosmopolitismo al restarle la visión de un gobierno único y considerar que podemos ser conciudadanos al preocuparnos por nuestros congéneres y no sólo por los que pertenecen a nuestra comunidad (Appiah 2008, pp. 15-16). El cosmopolitismo, afirma el anglo ghanés, es universalista: "cree que todos los seres humanos somos importantes y tenemos obligaciones compartidas de cuidarnos mutuamente [y] acepta el amplio abanico de la legítima diversidad humana" (Appiah 2008, p. 20). Lo importante, según esta idea, son las personas, así que para valorar a las culturas hay que considerar las consecuencias que éstas traen consigo. Este cosmopolitismo nos permite reconocer que somos falibles, es decir, que podemos equivocarnos y aprender de otros, en lugar de pretender convencer y convertir a toda la humanidad de una verdad o dogma, y mantiene la idea kantiana de que la dignidad de cada ser humano reside en su capacidad y su derecho de ejercer el autodominio (Appiah 2008, pp. 24-27). Finalmente, las palabras de Appiah convergen con el concepto de pluralismo que Kant definiera en su ApH como "aquel modo de pensar que consiste en no considerarse ni conducirse como encerrando en el propio sí mismo el mundo entero, sino como un simple ciudadano del mundo" (Ak. VII 130). Así, podemos concluir, junto con Appiah, que adoptar 
el pluralismo significa reconocer que existen muchos valores que valen la pena vivir, pero que no se puede vivir conforme a todos ellos (Appiah 2008, p. 33) y, si una cultura perjudica a las personas, el cosmopolita no tiene por qué tolerarla (Appiah 2008, p. 24), o bien, justificarla. 20

\section{Bibliografía}

Anderson-Gold, Sharon. 2001. Cosmopolitanism and Human Rights, Cardiff: University of Wales Press.

Appiah, Kwame Anthony. 2008. Mi cosmopolitismo, Buenos Aires: Katz.

Kant, Immanuel. 1991. Antropología en sentido pragmático, Madrid: Alianza.

2014. Antropología en sentido pragmático, México: FCEUAM-UNAM. 2003. Critica del discernimiento, Madrid: Machado Libros.

Ángel Porrúa.

2001. Crítica de la razón práctica, México: UAM-Miguel 2006. En defensa de la ilustración, Barcelona: Alba. . 2008. La metafisica de las costumbres, Madrid: Tecnos. . Kant on the web. Steve Palmquist's web site: http:// staffweb.hkbu.edu.hk/ppp/K1 texts.html (consultada el 13 de marzo de 2018).

Martiarena, Óscar. 2008. La formación del ciudadano del mundo, México: Alia.

Mendiola, Carlos. 2016. "La comunidad en el concepto de cosmopolitismo en Immanuel Kant” en Luis Guerrero Martínez. Ensayos para una hermenéutica de la filosofía, México: UIA.

Nussbaum, Martha. 2010. "Kant and cosmopolitanism" en Garrett Wallace Brown y David Held. The cosmopolitanism reader, Cambridge: Polity Press.

Rorty, Richard. 2000. "Derechos humanos, racionalidad y sentimentalismo" en Richard Rorty. Verdad y progreso, Barcelona: Paidos.

Wallace, R. Jay. 2006. Cómo argumentar sobre la razón práctica, MéXiCO: UNAM-IIF. 